\title{
Nuevas manifestaciones orales del síndrome branquio-óculo-facial. Caso clínico
} New oral manifestations of Branchio-oculo-facial syndrome. Case report

\author{
Dr. Juan B. García Flores ${ }^{a}$, Dr. César E. Escamilla Ocañas ${ }^{a}$ Dr. Héctor R. Martínez Menchaca ${ }^{a, b}$, \\ Dra. Ma. Guadalupe Treviño Alanís ${ }^{a}$ y Dr. Gerardo Rivera Silva ${ }^{a}$
}

\begin{abstract}
RESUMEN
El síndrome branquio-óculo-facial es una condición autosómica dominante con expresividad variable y que afecta particularmente las estructuras de la cara y cuello por un desarrollo inadecuado del primero y segundo arco branquial; presenta malformaciones de los ojos y oídos, con características faciales distintivas. Está asociado con alteraciones en el gen TFAP2A.

Se presenta una paciente de 9 años con fenotipo de síndrome branquio-óculo-facial y la presencia de dos nuevas manifestaciones orales, la úvula bífida y la lengua conhendidura central parcial, no descritas hasta ahora en esta condición clínica. Palabras clave: branquio-óculo-facial, arco branquial, úvula bífida, anomalías.
\end{abstract}

\begin{abstract}
The branchio-oculo-facial syndrome is a dominant autosomic condition with variable expressivity that affects particularly the facial and neck structures by an inadequate development of the first and second branchial arch. It is characterized by malformations of eyes and ears, with distinct facial characteristics. It is associated with alterations in TFAP2A gene. We present a patient with 9 years of age with phenotype of the branchio-oculo-facial syndrome and the presence of 2 new oral manifestations, the bifid uvula and the tongue with partial central cleft, not yet described in this clinical condition.

Key words: Branchio-oculo-facial, branchial arch, bifid uvula, tongue anomalies.
\end{abstract}

http:/ /dx.doi.org/10.5546/aap.2015.e14

\section{INTRODUCCIÓN}

El síndrome branquio-óculo-facial (BOF) es un trastorno autosómico dominante infrecuente con expresividad inconstante, caracterizado por alteraciones cutáneas y oculares, una apariencia facial distintiva por la presencia de parálisis facial

a. División de Ciencias de la Salud, Universidad de Monterrey, San Pedro Garza García, NL, MEX.

b. Department of Pediatric Dentistry, University of Louisville, School of Dentistry, Louisville, KY, USA.

Correspondencia:

Dr. Gerardo Rivera Silva: gerardo.rivera@udem.edu

Conflicto de intereses: ninguno que declarar.

Recibido: 15-4-2014

Aceptado: 4-6-2014 parcial, alteraciones del cabello y de los dientes. Fue descrito inicialmente por Fujimoto en $1987 .{ }^{1}$ Tiene una prevalencia de 1 caso por 1000000 nacidos vivos y afecta por igual a hombres y mujeres. La mayoría de los casos reportados son familiares. En el gen TFAP2A, se han identificado mutaciones en el sitio de empalme, deleciones iatrogénicas menores e inserciones. ${ }^{2}$ Este gen se encuentra localizado en el cromosoma 6. Las mutaciones de novo se detectan en el 50-60\% de los personas afectadas y las deleciones, en el $5 \%{ }^{3}$ Las deleciones más grandes del cromosoma 6p24-p25 que implican al gen TFAP2A son excepcionales. ${ }^{4}$

Esta condición está caracterizada clínicamente por la existencia de defectos branquiales cubiertos por piel normal, asociados con coloboma, microftalmia, obstrucción del conducto nasolagrimal, aparición de canas en forma prematura, hendiduras parciales o totales del labio superior, malformaciones auriculares y renales. Pueden existir otras malformaciones asociadas como las renales y más raramente las cardiopatías del tipo comunicación interatrial del tipo ostium secundum, tetralogía de Fallot y estenosis valvular pulmonar. En presencia de estas anomalías, la mortalidad puede incrementarse a más del $90 \%{ }^{5}$ Todo paciente con sospecha de este síndrome debe ser estudiado para excluir una probable heterogeneidad genética que se traduzca en diversas manifestaciones clínicas. ${ }^{6,7}$

El objetivo de este artículo es presentar un caso de una paciente con el síndrome de BOF, con dos manifestaciones clínicas que no han sido descritas, como son la úvula bífida y lengua con una hendidura central parcial.

\section{CASO CLÍNICO}

Niña de 5 años de edad que presenta una historia de 3 años con trastornos del lenguaje del tipo disartria. Producto del primer embarazo y con peso al nacer de 2850 gramos. Como antecedentes personales patológicos, presentó una parálisis parcial del nervio facial desde los 20 meses de edad y una intervención para la 
reparación de paladar hendido a los 24 meses de edad. Se había realizado diagnóstico previo de síndrome de Pierre Robin. No había antecedentes de fenotipo sospechoso en los padres; hija única. En la exploración física, se encontró talla: $101 \mathrm{~cm}$ (centilo 3); PC: $48 \mathrm{~cm}$ (centilo 10); peso: $15 \mathrm{~kg}$ (centilo 10); presencia de algunas canas, lesiones faciales eritematosas y hemangiomatosas; hipertelorismo discreto; orejas anormales y con implantación baja; asimetría facial provocada por una parálisis parcial del séptimo par craneal (Figura 1A); atresia del conducto nasolagrimal izquierdo y habla hipernasal. Asimismo, el examen oral reveló úvula bífida (Figura 1. $B$.$) , lengua corta con una hendidura central$ parcial (Figura 1. B., C.), ausencia de incisivos centrales superiores deciduos y cicatriz del cierre quirúrgico del paladar.

Los hallazgos radiográficos bucales mostraron las piezas dentarias permanentes sin erupcionar y se corroboró la ausencia de incisivos centrales superiores primarios. El cariotipo prenatal resultó normal 46, XX. La secuenciación en el gen TFAP2A reveló la mutación c.763A>G
(p.Arg255Gly). El ecocardiograma y el ultrasonido renal fueron reportados sin alteraciones.

La paciente fue tratada con el apoyo de un grupo multidisciplinario de especialistas, que incluyó odontólogos, genetistas, terapistas del lenguaje, cirujanos plásticos y cráneo-faciales.

\section{COMENTARIO}

El cuadro clínico del síndrome BOF se caracteriza por ser variable ya que va a depender de las variaciones génicas presentes. ${ }^{8}$ El diagnóstico, en este caso, se basó en los antecedentes de corrección quirúrgica del paladar hendido, la disartria provocada por la parálisis facial parcial, lesiones eritematosas de la piel, atresia del conducto nasolagrimal izquierdo, hipertelorismo, orejas malformadas, presencia de algunas canas y ausencia de los incisivos centrales superiores. Ante la evidencia diagnóstica del síndrome BOF, se realizó análisis por secuenciación, que reveló la mutación c.763A>G (p.Arg255Gly) en el gen TFAP2A. ${ }^{9}$ Más del $95 \%$ de los pacientes en quienes se realiza el diagnóstico del síndrome BOF tienen una variante

FIGURA 1. Imagen fotográfica frontal de la paciente, en la que se detectan algunas canas, orejas anormales y con implantación baja, hipertelorismo, asimetría facial provocada por parálisis parcial de séptimo par craneal y lesiones faciales eritematosas (A); fotografía intraoral que muestra úvula bífida y lengua con una hendidura central parcial (B); imagen frontal de la lengua que pone en evidencia que es corta, con una hendidura central parcial e hipertrofia de las papilas gustativas (C)
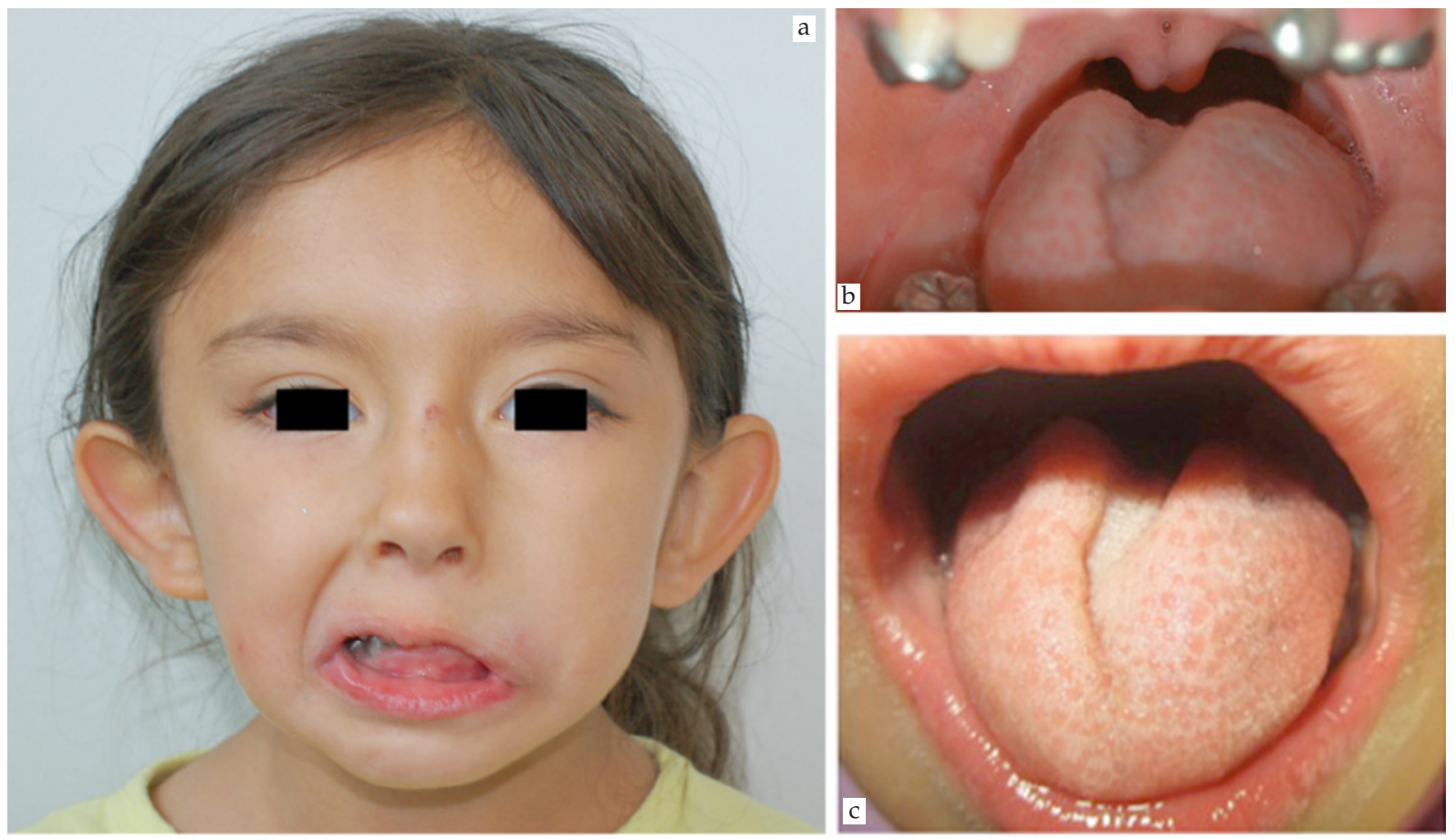
en la secuencia del gen TFAP2A. ${ }^{10,11}$ Además de lo mencionado con antelación, en este caso, se destacó la presencia de úvula bífida y lengua con una hendidura central parcial, manifestaciones clínicas que no han sido reportadas; sin embargo, por su origen embrionario en la región del piso faríngeo, son compatibles como hallazgos clínicos de este padecimiento. ${ }^{12}$

El diagnóstico diferencial se estableció con el síndrome branquio-oto-renal, el cual se caracteriza por fístulas o quistes branquiales, hipoplasias de pabellones auriculares, sordera y alteraciones renales, (duplicación, desplazamiento, hipoplasia o agenesia) $;{ }^{13}$ con el síndrome de Cohen caracterizado por alteraciones dentales, retraso en el desarrollo del habla, microcefalia, miopía, neutropenia y obesidad troncal; y finalmente con el síndrome de CHARGE, que presenta coloboma ocular, parálisis facial, alteraciones cardíacas, atresia de coanas, retraso en el crecimiento y desarrollo, hipoplasia genital, orejas anormales y sordera. ${ }^{14,15}$ En nuestro caso, el pronóstico fue bueno ya que no presentó alteraciones renales ni cardíacas.

El síndrome BOF representa un reto diagnóstico y, aunque existe el análisis por secuenciación para identificar alteraciones en el gen TFAP2A, este procedimiento no está disponible en todos los centros de atención. Se debe orientar a los progenitores ante el diagnóstico y considerar un tratamiento multidisciplinario.

\section{REFERENCIAS}

1. Fujimoto A, Lipson M, Lacro RV, Shinno NW, et al. New autosomal dominant branchio-oculo-facial syndrome. Am J Med Genet 1987;27(4):943-51.
2. MilunskyJM,MaherTA,ZhaoG, Roberts AE, etal.TFAP2A mutations result in branchio-oculo-facial syndrome. Am J Hum Genet 2008;82(5):1171-7.

3. Milunsky JM, Maher TM,Zhao G, Wang Z, et al. Genotypephenotype analysis of the branchio-oculo-facial syndrome. Am J Med Genet A 2011;155A(1):22-32.

4. Misceo D, BjorgoK, OrmerodE, Ringen O, etal. A denovo6p interstitial deletion and a complex translocation involving chromosomes 2, 6, and 14 in a mildly developmentally delayed patient. Am J Med Genet A 2008;146A(24):3230-3.

5. Machado-Rosa RF, Gazzola-Zen PR, Graziadio C, Paskulin, GA. Síndrome brânquio-óculo-facial(BOFS) ecardiopatias congênitas. Arq Bras Cardiol 2009;92(2):e33-5.

6. Verret DJ, Murray AD, Hobar PC. Branchio-oculo-facial syndrome with ectodermal parthyroid tissue. Otolaryngol Head Neck Surg 2005;133(6):983-4.

7. Raveh E, Papsin BC, Forte V. Branchio-oculo-facial syndrome. Int J Pediatr Otorhinolaryngol 2000;53(2):149-56.

8. Galliani E, Burglen L, Kadlub N, Just W, et al. Craniofacial phenotype in the branchio-oculo-facial syndrome: four case reports. Cleft Palate Craniofac J 2012;49(3):357-64.

9. Reiber J, Sznajer Y, Posteguillo EG, Müller D, et al. Additional clinical and molecular analyses of TFAP2A in patients with the branchio-oculo-facial syndrome. Am J Med Genet A 2010;152A(4):994-9.

10. Tekin M, Sirmaci A, Yüksel-Konuk B, Fitoz S, et al. A complex TFAP2A allele is associated with branchio-oculofacial syndrome and inner ear malformation in a deaf child. Am J Med Genet A 2009;149A(3):427-30.

11. Al-Dosari MS, Almazyad M, Al-Ebdi L, Mohamed JY, et al. Ocular manifestations of branchio-oculo-facial syndrome: report of a novel mutation and review of the literature. $\mathrm{Mol}$ Vis 2010;16:813-8.

12. Abbo O, Bieth E, Ballouhey Q, Vaysse F, et al. Branchioculo-facial syndrome: a case report to highlight recent genetic considerations. J Plast Reconstr Aesthet Surg 2012;65(11):1573-5.

13. Trummer T, Müller D, Schulze A, Vogel W, et al. Branchiooculo-facial syndrome and branchio-otic/branchiooto-renal syndromes are distinct entities. J Med Genet 2002;39(1):71-3.

14. FrascariF, BiethE, GalinierP, Just W, etal.Syndromebrachiooculo-facial. Ann Dermatol Venereol 2012;139(8-9):550-4.

15. Park MY, Kim YC. A case of branchio-oculo-facial syndrome. Ann Dermatol 2009;21(3):288-90. 